\title{
Medições da rigidez do solo para previsão da estabilidade da camada de cobertura de um aterro sanitário
}

\section{Soil Rigidity Measurements for a Stability Estimate of a Landfill Cover Layer}

\author{
Mediciones de la Rigidez del Suelo para Previsión de la \\ Estabilidad de la Capa de Cobertura de un Vertedero
}

\section{Mesures de la rigidité du sol pour prévoir la stabilité de la couche extérieure d'un site d'enfouissement}

Samara Castro Freire iD sosamara505@gmail.com Universidade Federal do Ceará (UFC)

Francisco Carlos Henrique Pio de Oliveira iD franciscocarloshpo@gmail.com Instituto Federal do Ceará (IFCE)

Eliana Dantas Ribeiro (iD elianadtr@hotmail.com Instituto Federal do Ceará (IFCE)

\section{Roberto Antônio Cordeiro} da Silva iD roberto@det.ufc.br Universidade Federal do Ceará (UFC)

Gemmelle Oliveira Santos (iD gemmelle@ifce.edu.br Instituto Federal do Ceará (IFCE)

\section{Resumo}

Esta pesquisa utilizou um equipamento chamado Humboldt GeoGauge ${ }^{\mathrm{TM}}$, muito aplicado na avaliação da qualidade da compactação dos solos em obras de engenharia civil, para medir a rigidez da camada de cobertura (bermas e talude) de uma parte do maior aterro sanitário do estado do Ceará, em processo de encerramento da sua vida útil. Foram escolhidos nove locais, sendo três na berma inferior, três no talude e três na berma superior. Em cada ponto foram extraídas três leituras da rigidez do solo e do módulo de deformação para obtenção de uma média. Os resultados mostraram que as bermas são mais rígidas que o talude, indicando uma maior e menor compactação do solo, respectivamente. Cinco pontos foram classificados como fracamente rígidos e os outros quatro pontos, como rígidos. Tais resultados foram discutidos no contexto de outros importantes parâmetros do aterro estudado: teor de umidade do solo e dos resíduos; presença de gases; permeabilidade e granulometria dos solos; e presença ou ausência de fissuras/trincas sobre a cobertura, considerando diversas abordagens da literatura. O Humboldt GeoGauge ${ }^{\mathrm{TM}}$ mostrou-se extremamente viável e adequado para a medição da rigidez dos solos da camada de cobertura do aterro sanitário em questão, abrindo, inclusive, novas linhas de investigação para a engenharia sanitária e ambiental e, principalmente, para o controle geotécnico desses empreendimentos.

Palavras-chave: Humboldt GeoGauge ${ }^{\mathrm{TM}}$. Compactação dos solos. Rigidez dos solos. Cobertura de aterros sanitários.

\footnotetext{
Abstract

This study used an equipment called Humboldt GeoGauge ${ }^{\mathrm{TM}}$, commonly applied in the evaluation of the quality of soil compaction in civil engineering works, to measure the rigidity of the cover layer (berms and slope) of a part of the largest Landfill of the State of Ceará, in the process of closing its useful life. Nine sites were selected, three on the lower berm, three on the slope and three on the upper berm. At each point three readings of the soil rigidity and the strain modulus were extracted to obtain a mean. The results revealed that the berms are more rigid than the slope, indicating a greater and smaller soil compaction, respectively. Five points were classified as weakly rigid and the other four points were rigid. These results were discussed in the context of other important parameters of the landfill studied: soil and residue moisture content, presence of gases, soil permeability and granulometry, presence or absence of fissures/cracks on the cover, considering several approaches in the literature. The Humboldt GeoGauge ${ }^{\mathrm{TM}}$ proved to be extremely feasible and suitable for measuring the soil rigidity of the cover layer of the landfill in question, opening up new lines of research for
} 
sanitary and environmental engineering and especially for geotechnical control of these enterprises.

Keywords: Humboldt GeoGauge ${ }^{\mathrm{TM}}$. Soil compaction. Soil rigidity. Landfill cover.

\section{Resumen}

Esta investigación utilizó un aparato llamado Humboldt GeoGauge ${ }^{\mathrm{TM}}$, muy aplicado en la evaluación de la calidad de compactación de los suelos en obras de ingeniería civil, para medir la rigidez de la capa (bermas y talud) de una parte del más grande Vertedero del Estado del Ceará, en proceso de desactivación. Fueron elegidos nueve sitios: tres en la berma inferior; tres en el talud y tres en la berma superior. En cada punto fueron extraídas tres lecturas de la rigidez del suelo y del módulo de deformación para obtención de una media. Los resultados demostraron que las bermas son más rígidas que el talud, indicando una mayor y una menor compactación, respectivamente. Cinco puntos fueron clasificados como rigidez débil y los otros cuatro puntos como rígidos. Tales resultados fueron discutidos en el contexto de otros importantes parámetros del vertedero estudiado: porcentaje de humedad del suelo y de los desechos, presencia de gases, permeabilidad y granulometría de los suelos, presencia o ausencia de grietas/hendiduras sobre la cobertura, considerando distintos enfoques de la literatura. El Humboldt GeoGauge ${ }^{\mathrm{TM}}$ demostró ser extremamente viable y adecuado para la medición de la rigidez de los suelos de la capa de cobertura del vertedero en cuestión, abriendo, incluso, nuevas líneas de investigación para la ingeniería sanitaria y ambiental y, principalmente, para el control geotécnico de estos emprendimientos.

Palabras-clave: Humboldt GeoGauge ${ }^{\mathrm{TM}}$. Compactación de los suelos. Rigidez de los suelos. Cobertura de vertederos.

\section{Résumé}

Cette recherche a utilisé un équipement appelé Humboldt GeoGauge ${ }^{\mathrm{TM}}$, largement utilisé pour évaluer la qualité du compactage du sol dans les travaux de génie civil, afin de mesurer la rigidité de la couche extérieure (bas-côtés et pente) d'une partie du plus grand site d'enfouissement sanitaire de l'État du Ceará, en train de fermer sa vie utile. Neuf sites ont été choisis, trois dans le bas-côté inférieur, trois dans la pente et trois dans le bas-côté supérieur. À chaque point, trois lectures de la rigidité du sol et du module de déformation ont été extraites pour obtenir leurs moyennes. Les résultats ont montré que les bas-côtés sont plus rigides que la pente, cela indique un plus grand et un plus petit compactage du $\mathrm{sol}$, respectivement. Cinq points ont été classés comme faiblement rigides et les autres quatre comme rigides. Ces résultats ont été discutés dans le contexte d'autres paramètres importants du site étudié: taux d'humidité du sol et des résidus, présence de gaz, perméabilité du sol et granulométrie, présence ou absence de fissures sur la couche en considérant

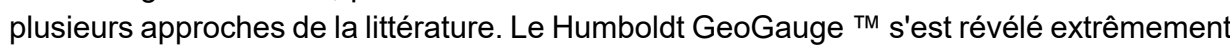
viable et adéquat à la mesure de la rigidité du sol de la couche d'enfouissement. En fait, il a permis les ouvertures des nouvelles lignes de recherche pour la génie sanitaire et environnemental, en particulier, pour le contrôle géotechnique de ces entreprises.

Mots-clés: Humboldt GeoGauge ${ }^{\mathrm{TM}}$; Compactage du sol. Rigidité du sol. Couche des sites d'enfouissement.

\section{Introdução}

Os aterros sanitários são obras de engenharia destinadas ao armazenamento seguro de resíduos sólidos urbanos (RSU) que não sofreram um tratamento mais nobre (reutilização, reciclagem, compostagem, aproveitamento energético etc.). 
No Brasil, os aterros sanitários são o único método de disposição de RSU sobre o solo reconhecido pela Lei n. ${ }^{\circ} 12.350$ (BRASIL, 2010), que trata da Política Nacional de Resíduos Sólidos, e devem ser projetados para minimizar impactos ambientais e sociais.

O crescimento das cidades somado à diminuição de áreas aptas para instalação de novos aterros sanitários (em termos técnicos, econômicos e ambientais) representam grandes entraves, contribuindo para a adoção de medidas de ampliação da vida útil dos empreendimentos em operação e para a reutilização/ alteamento de aterros encerrados.

Para Law et al. (2013), a expansão vertical de aterros sanitários exige avaliação detalhada do local com relação à estabilidade das encostas, à capacidade de carga da fundação e à qualidade dos sistemas de drenagem de lixiviados. É fator-chave o conhecimento sobre a história da construção do aterro sanitário (LOPES; GOMES, 2013).

Conforme Kölsch e Bauer (2009), assegurar a estabilidade é questão geotécnica central durante a operação e encerramento dos aterros sanitários, sendo que a referida estabilidade depende de vários parâmetros, quais sejam: nível de lixiviado; coesão e ângulo de atrito dos resíduos (RONG; ZHAOGUI; TUGEN, 2011); composição e compactação dos resíduos; condições climáticas; geometria do aterro; estabilidade do solo (KÖLSCH; BAUER, 2009). Depende, também, do comportamento dos recalques dos resíduos e da integridade da camada de base e de cobertura (LAW et al., 2013).

Para Saravanan et al. (2007), do ponto de vista geotécnico, a seleção do tipo de camada depende da inclinação dos taludes, da altura do aterro, das propriedades que existem na interface solo-resíduo e do comportamento das deformações horizontais. Segundo os autores, a camada precisa resistir, também, à sobreposição de novos resíduos, à expansão/contração térmica de materiais e à transferência de tensões.

As camadas de cobertura dos aterros sanitários estão sujeitas, ainda, a um grande número de solicitações: variações de temperatura; ciclos de umedecimento e secagem; erosão por água ou vento; presença de raízes e animais (BOSCOV, 2008). A própria degradação da matéria orgânica agrega riscos geotécnicos em função da formação de depressões e deslocamentos de solo da cobertura final para o interior dos resíduos, ocasionando a perda de estanqueidade e o surgimento de fissuras e trincas (ALVES, 2010), o que prejudica a selagem do aterro e sua consequente sustentabilidade (COSTA, 2015).

As fissuras podem resultar em caminhos preferenciais de fluxos de gases (JONES; DIXON, 2005) e infiltração de grandes volumes de águas pluviais para o interior da massa de resíduos, ocasionando deformações do maciço e, consequentemente, deslizamento da camada (XU; TOLAYMANT; TOWSEND, 2012; PENG et al., 2016; FENG; GAO, 2012). Como o RSU é altamente deformável, as rupturas são muito grandes e outros materiais presentes no aterro (solos, sistemas de drenagem de lixiviados e gases, e coberturas) podem não resistir (LOPES; GOMES, 2013).

O comportamento geotécnico dos aterros sanitários requer também o conhecimento sobre as características físicas, químicas, mecânicas e sobre as propriedades hidráulicas dos resíduos sólidos urbanos e sua variação ao longo do tempo, por isso os recalques de resíduos são caracterizados por fases distintas, cujos mecanismos são mais numerosos e complexos que os do solo (LOPES; GOMES, 2013).

Os aterros sanitários - ao contrário dos maciços de solos compactados - são constituídos por diferentes tipos de resíduos, que interagem e formam um maciço heterogêneo e poroso com comportamento peculiar, cuja composição dos constituintes e sua degradação com o passar do tempo promovem grandes e diferentes variações volumétricas no interior do aterro, resultando em recalques diferenciais e significativos (SILVA et al., 1998).

Diante de tantas variáveis, calcular corretamente a estabilidade de um aterro sanitário é uma tarefa ambiciosa, mas que tem despertado - sob diversas metodologias - o interesse de pesquisadores do Brasil (STRAUSS, 1998; OLIVEIRA, 2002; CATAPRETA, 2008; BORGATTO, 2010; SCHULER, 2010; MOREIRA et al., 2013; SILVA, 2014; REMÉDIO, 2014; DA SILVA, 2017) e do mundo (JIANGUO; YONGFENG; QINGZHONG, 2001; HOSSAIN; HAQUE, 2009; XU; TOLAYMANT; TOWSEND, 2012; BABU; REDDY; SRIVASTAVA, 2014). 
As falhas nos sistemas de cobertura de aterros encerrados e seus respectivos acidentes geotécnicos vêm sendo estudadas na literatura (KOERNER; HWU, 1991; ALBRIGHT, 2006; HUVAJ-SARIHAN; STARK, 2008; EID, 2011; YIN et al., 2016), mas a maioria das publicações científicas não detalha os equipamentos utilizados, os métodos de instalação e as principais dificuldades encontradas (LOPES; GOMES, 2013). Assim, esta pesquisa ajuda a reduzir essa lacuna da literatura ao trazer resultados de um monitoramento in situ de uma camada de cobertura de um aterro sanitário que se encontra em processo de desativação.

Atualmente, o Brasil abriga 687 aterros sanitários, sendo que $61 \%$ possui alguma atividade classificada como de monitoramento ambiental (Sistema Nacional de Informações Sobre Saneamento - SNIS, 2016). No estado do Ceará existem seis aterros sanitários (SNIS, 2016), sendo que o maior deles fica na Região Metropolitana de Fortaleza: o Aterro Sanitário de Caucaia (ASC), com 123 hectares.

O ASC começou a receber os resíduos sólidos de Fortaleza em 1998 e deve encerrar suas atividades este ano (2019), sendo de extrema importância iniciar seu monitoramento geotécnico. Os resultados obtidos, além de localmente inéditos, podem aprimorar a rotina operacional e o monitoramento geotécnico dos demais aterros sanitários do estado do Ceará.

\section{Objetivos}

\subsection{Geral}

Determinar as condições de estabilidade geotécnica das camadas de cobertura de uma área do Aterro Sanitário de Caucaia-CE

\subsection{Específicos}

Medir a rigidez do solo da camada de cobertura do aterro;

Comparar a rigidez entre bermas e taludes;

Classificar, diante da literatura, as camadas de cobertura quanto à estabilidade;

Contribuir para a redução da lacuna na literatura relativa ao tema do monitoramento in situ de aterros sanitários.

\section{Metodologia}

O trabalho foi desenvolvido no Aterro Sanitário de Caucaia (ASC), que recebe aproximadamente 4.500 toneladas de resíduos sólidos urbanos por dia e dista $30 \mathrm{Km}$, pelas BR-222 ou BR-020, de Fortaleza-CE.

Em relação à tipologia, $46 \%$ (em massa) do que chega ao ASC tem origem domiciliar, $17 \%$ são provenientes dos pontos de lixo cadastrados no sistema de monitoramento da Prefeitura, $14 \%$ são resíduos da atividade de capina, $11 \%$ são resíduos da construção e demolição provenientes de pequenos geradores (utilizado em camadas intermediárias quando a disponibilidade de solo encontra-se reduzida), $7 \%$ são classificados como resíduos de particulares (provenientes de empresas em geral), 3\% são resíduos de poda (submetidos a trituração para fabricação de briquetes) e $2 \%$ são resíduos de varrição.

Em 2018, a análise de 32 amostras de $100 \mathrm{Kg}$ (cada) de resíduos sólidos domiciliares destinados ao ASC mostrou que, aproximadamente, $34,20 \%$ são resíduos classificados como orgânicos (especialmente restos de alimentos), 39,20\% são passíveis de reciclagem e $26,60 \%$ são rejeitos (fraldas usadas, areia etc.). A Figura 1 mostra o local onde os ensaios foram realizados no contexto da área do ASC. 
Figura 1: Limites do Aterro Sanitário de Caucaia (em amarelo) e localização da área pesquisada (em azul).

a)

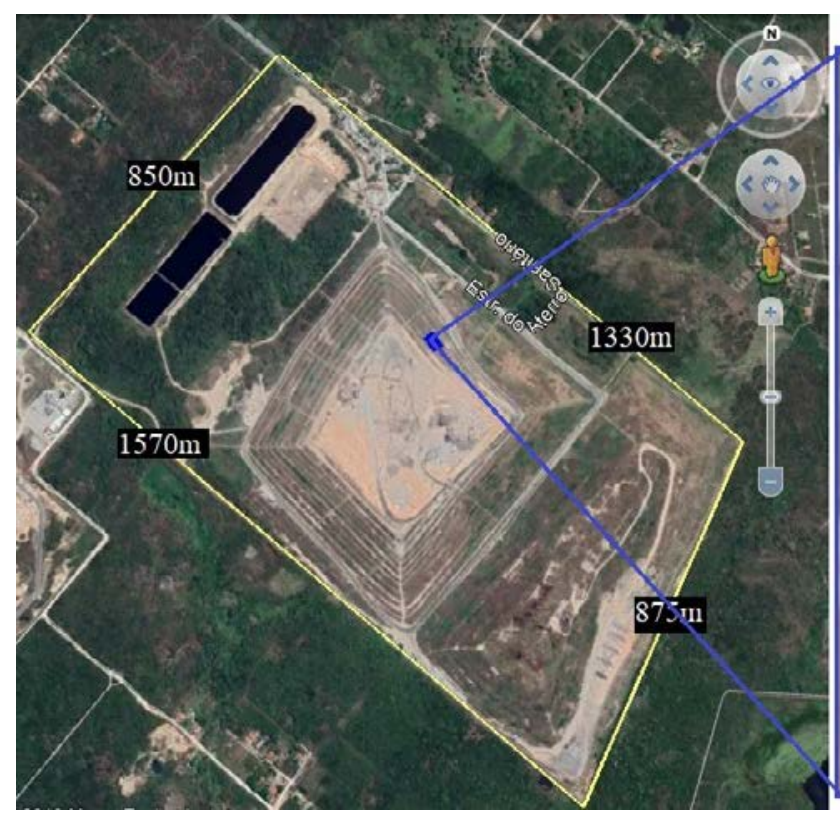

b)

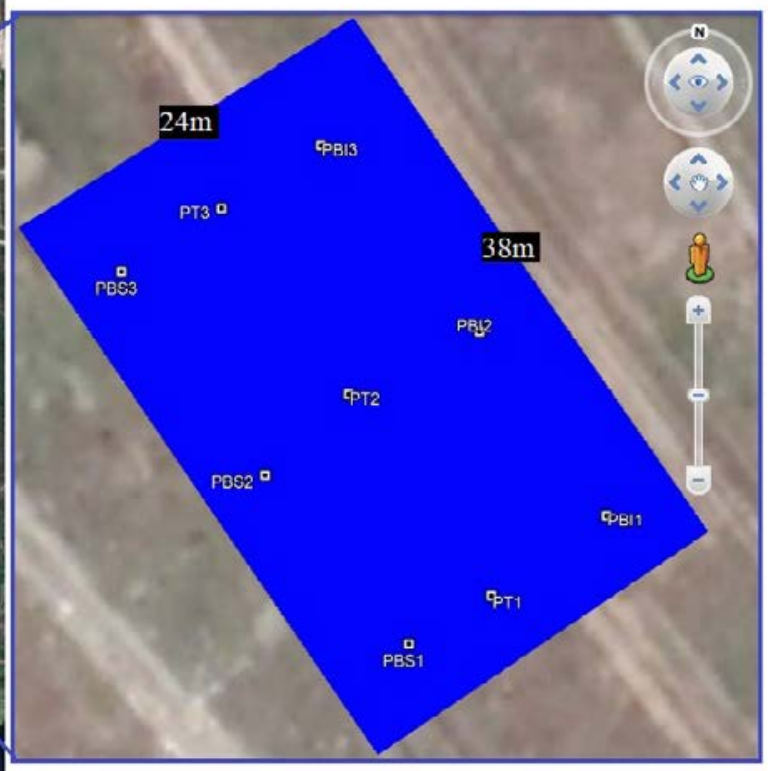

Fonte: Adaptado do Google, 2018.

A área pesquisada tem $929 \mathrm{~m}^{2}$ e $125 \mathrm{~m}$ de perímetro. Foi composta por 02 bermas e 01 talude. Seus limites são apresentados na Tabela 1. Foram analisados 09 pontos e em cada ponto foram extraídas 03 medições para obtenção de uma média Fig.2.

Tabela 1: Coordenadas geográficas dos limites da área pesquisada.

\begin{tabular}{c|c|c|c|c|c}
\hline Pontos & Latitude (S) & Longitude (O) & Pontos & Latitude (S) & Longitude (O) \\
\hline A & $3^{\circ} 47.632^{\prime}$ & $38^{\circ} 40.308^{\prime}$ & C & $3^{\circ} 47.615^{\prime}$ & $38^{\circ} 40.321^{\prime}$ \\
B & $3^{\circ} 47.625^{\prime}$ & $38^{\circ} 40.298^{\prime}$ & D & $3^{\circ} 47.608^{\prime}$ & $38^{\circ} 40.310^{\prime}$ \\
\hline
\end{tabular}

Fonte: Elaborado pelos autores, 2018.

Figura 2: Distribuição dos pontos analisados na área estudada.

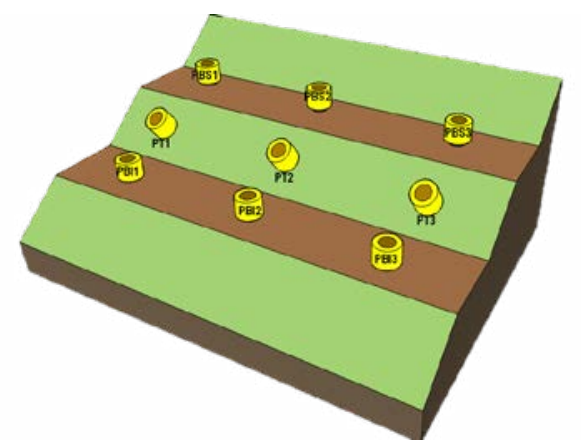

Fonte: Elaborado pelos autores, 2018.

Legenda:

PBS=Ponto na berma superior

$\mathrm{PBI}=$ Ponto na berma inferior

$\mathrm{PT}=$ Ponto no talude 
A rigidez do solo da camada de cobertura foi medida com um equipamento chamado Humboldt GeoGauge $^{\mathrm{TM}}$. Ele permite um ensaio não destrutivo em comparação a outros ensaios comumente realizados no dimensionamento mecanístico-empírico do solo (MAIA; SILVA; BARROSO, 2018), e consta na Fig.3. Os resultados obtidos por Abu-Farsakh et al. (2004) sugerem que o Humboldt GeoGauge ${ }^{\mathrm{TM}}$ pode ser uma ferramenta promissora para medir in situ da rigidez dos materiais e aterros, sendo fundamental evitar sua colocação sobre solos com visíveis trincas decorrentes da contração natural (ALSHIBLI et al., 2005).

Figura 3: Vista do equipamentoHumboldt GeoGauge $^{\text {TM }}$ sobre o solo do aterro sanitário.

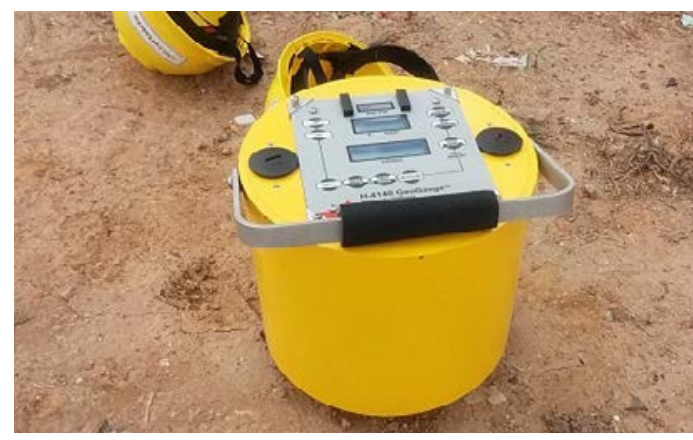

Fonte: Elaborado pelos autores, 2018.

O Humboldt GeoGauge ${ }^{\mathrm{TM}}$ é um equipamento eletromecânico e portátil, que in situ traduz a rigidez de camadas compactadas de solo ou agregados. O equipamento pesa cerca de $10 \mathrm{Kg}$ e tem forma cilíndrica, com 0,280 m de diâmetro e 0,254 m de altura. O seu apoio sobre a superfície do terreno é feito a partir de uma base anelar metálica. O contato entre a base e superfície deve ser de, no mínimo, 60\%, para evitar erros nas leituras (CONDE; CALDEIRA; LOPES, 2010).

O Humboldt GeoGauge ${ }^{\mathrm{TM}}$ aplica uma força dinâmica muito reduzida, com frequências variáveis (com incrementos de $4 \mathrm{~Hz}$ ) entre 100 e $196 \mathrm{~Hz}$ (ALSHIBLI et al., 2005). Durante o ensaio, a força aplicada (F) e o respectivo deslocamento $(\delta)$ são medidos para 25 diferentes frequências de vibração. A força máxima produzida pelo Humboldt GeoGauge ${ }^{\mathrm{TM}}$ corresponde a $9 \mathrm{~N}$ e os deslocamentos induzidos no solo não ultrapassam 1,27x10-6 m (ALSHIBLI et al., 2005).

Como resultado do ensaio, o equipamento fornece o valor médio da rigidez (conforme a seguinte equação: $k=F / \delta$ ) medidos para cada frequência, e cada ensaio tem uma duração aproximada de 1,5 minutos. Para cada frequência, o equipamento faz a medição e a comparação de velocidades de dois sensores. Os transdutores utilizados são geofones que, pelo seu princípio de funcionamento, fornecem uma variação de voltagem proporcional à velocidade. Tendo em conta as características do ensaio, este tipo de sensores são suficientemente precisos e constituem uma alternativa mais econômica em relação à utilização de acelerômetros (HUMBOLDT, 2007; ALSHIBLI et al., 2005; FORTUNATO, 2005).

A diferença entre os resultados obtidos com o Humboldt GeoGauge ${ }^{\mathrm{TM}}$ e um ensaio de carga com placas não ultrapassa 10\% (NELSON; SONDAG, 1999). Tal diferença é ainda menor se comparada com os resultados de programas de computador: 5\% (LENKE; GORDON McKEEN; GRUSH, 2003). Esses são os valores de referência.

Conforme Lenke, Gordon McKeen e Grush (2003), a rigidez do solo, indiscutivelmente, é o parâmetro de engenharia mais importante no controle da compactação e resistência mecânica dos solos. A estabilidade do aterro sanitário mantém relação com a rigidez dos solos. Geralmente, a rigidez diminui com o crescimento do fissuramento (LAKSHMIKANTA, 2009), a presença de uma pressão negativa nos poros (FREDLUND; RAHARDJO, 1993), o aumento do teor de umidade (NELSON; SONDAG, 1999), problemas de drenagem 
interna de lixiviados e externa de águas pluviais (QUINTA-FERREIRA, 2015) e acúmulo de gás no interior do aterro (MERRY; KAVAZANJIAN; FRIT, 2005).

\section{Resultados e discussão}

Os valores de rigidez foram maiores nas bermas do que no talude, indicando - em primeira análise uma maior e menor compactação dos solos, respectivamente Tab.(2). A própria inclinação do talude e as dificuldades que se tem para sua execução (dentro da atribulada rotina de um aterro sanitário) ajudam a entender seus resultados. Além disso, são as bermas que sofrem constante compactação devido ao tráfego de veículos e máquinas pesadas que buscam acessar a frente de operação (setor de descarga) do aterro sanitário, justificando seus valores maiores.

Tabela 2: Valores da rigidez e do módulo de deformação medidos nas bermas e talude.

\begin{tabular}{c|c|c|c}
\hline Local & Pontos & Rigidez $(\mathrm{MN} / \mathrm{m})$ & Módulo de deformação $(\mathrm{MPa})$ \\
\hline \multirow{3}{*}{ Berma inferior } & PBI 1 & 12,1 & 104,9 \\
& PBI 2 & 8,4 & 72,8 \\
& PBI 3 & 10,1 & 87,2 \\
\hline \multirow{3}{*}{ Talude } & PT 1 & 2,8 & 24,2 \\
& PT 2 & 1,5 & 12,7 \\
& PT 3 & 2,1 & 63,1 \\
\hline \multirow{3}{*}{ Berma superior } & PBS 1 & 7,4 & 93,6 \\
& PBS 2 & 10,8 & 93,3 \\
\hline
\end{tabular}

$\mathrm{PBI}=$ Ponto na berma inferior, $\mathrm{PT}=$ Ponto no talude, $\mathrm{PBS}=$ Ponto na berma superior

Fonte: Elaborado pelos autores, 2018.

Para valores de rigidez abaixo de $10 \mathrm{MN} / \mathrm{m}$ (medidas com o Humboldt GeoGauge ${ }^{\mathrm{TM}}$ ), Chen et al. (1999) classificam a compactação do solo como fracamente rígida. Nesta pesquisa, cinco pontos teriam esse conceito: o PBI 2, os três pontos do talude e o PBS 1. Valores de rigidez acima de $10 \mathrm{MN} / \mathrm{m}$ até $12,96 \mathrm{MN} / \mathrm{m}$, conforme Collins e Connor (2011), correspondem a compactação como rígida, ocorrendo em quatro pontos desta pesquisa: PBI 1 e 3, PBS 2 e 3). Nenhum ponto da área estudada foi classificado como altamente rígido (acima de 14,82 MN/m, conforme definição de White et al., 2005).

Na berma inferior e no talude, a rigidez foi menor no "ponto central" (PBI 2 e PT 2), indicando a ausência de cuidados no fechamento das escavações realizadas pelo operacional do aterro sanitário quando da instalação de drenos horizontais de captação forçada de gás (na realidade, observou-se que, para evitar danos aos tubos de PEAD, Fig.4, pouca ou nenhuma compactação tem sido realizada sobre esse local). Conforme Fredlund e Rahardjo (1993), a presença, em solos, de uma pressão negativa nos poros (sucção) é um dos principais fatores de alteração do comportamento geomecânico.

Conforme Nelson e Sondag (1999), uma menor rigidez pode ter relação com um teor de umidade inadequado para a efetiva compactação do solo, sem descartar a possibilidade de o próprio compactador soltar o solo mais superficial, causando uma leve diminuição na rigidez. Para Quinta-Ferreira (2015), os menores valores da rigidez estão associados às piores áreas, ou seja, locais em que o grau de degradação da camada é maior. Além disso, explica o autor, baixo módulo de rigidez em áreas significativas indica grave problema de drenagem.

Os resultados obtidos em uma pesquisa paralela indicam uma maior concentração de gases próximo ao "centro" da área estudada (PBI 2 e PT 2), ajudando a entender que a pressão exercida pelo acúmulo desses gases sob a camada de cobertura provavelmente está aumentando a instabilidade do solo e diminuindo sua rigidez. 
Figura 4: Escavação e instalação dos drenos horizontais de PEAD de 160 mm.

b)

a)
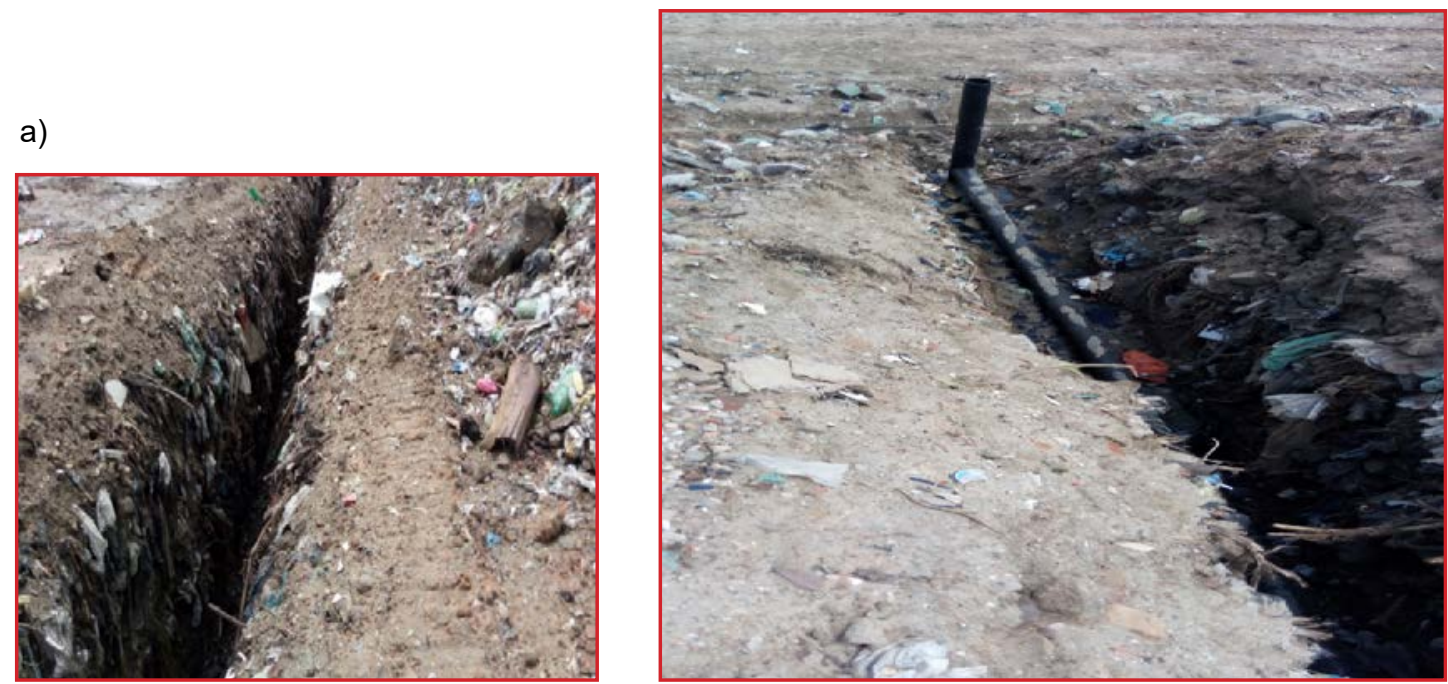

Fonte: Elaborado pelos autores, 2018.

Merry, Kavazanjian e Fritz. (2005) lembram que o acúmulo de gás no aterro pode gerar uma pressão excessiva entre os poros, entre o solo e os resíduos, diminuindo o fator de segurança referente à estabilidade do talude. Pressões excessivas de gás reduzem ainda mais a tensão efetiva dos resíduos e a resistência ao cisalhamento (JAFARI; STARK; MERRY, 2013).

Conforme a literatura (PESTANA, 2008; CONDE; CALDEIRA; LOPES, 2010; CONDE et al., 2013), existe uma significativa correlação da rigidez medida com o Humboldt GeoGauge ${ }^{\mathrm{TM}}$ e o teor de água, ou seja, o aumento do teor de água implica na diminuição da rigidez do solo. Um solo que se encontra saturado possui pouca resistência (FREDLUND; RAHARDJO, 1993).

Os ensaios de compactação do solo (Norma Brasileira - NBR 7182/1986) realizados com amostras da área pesquisada mostraram umidade ótima, variando entre 13,0 e 16,6\%, e massa específica aparente de seca máxima, entre 1,731 e 1,840 $\mathrm{g} / \mathrm{cm}^{3}$. Estes resultados são semelhantes aos de Santos (2012), que pesquisou o mesmo aterro sanitário sob os mesmos ensaios. Por outro lado, foi verificado um déficit de compactação na camada de cobertura, variando entre 10,6 e 16,0\% (déficit = diferença entre a compactação existente a padrão/energia de proctor normal).

O solo da área estudada foi classificado como areno-silto-argiloso, relativamente bem graduado e com grande \% de areia, que geralmente tem mais atrito que coesão. Ainda sob tal condição, os resultados obtidos com o Humboldt GeoGauge ${ }^{\mathrm{TM}}$, em termos técnicos, podem ser considerados adequados (QUINTA-FERREIRA; ANDRADE, 2008).

Os teores de umidade da mistura solo-resíduo de cinco amostras extraídas do local escavado para disposição do dreno mostrado na Figura 4 apontaram para teores variando entre 10,29 - 16,34\% de umidade, resultados semelhantes aos obtidos por Medeiros et al. (2002) em outros aterros sanitários do Brasil.

A presença de água, por sua vez, tem relação com a permeabilidade do solo. O solo da área estudada tem uma permeabilidade que varia entre $1,4 \times 10^{-4}$ e $4,5 \times 10^{-3} \mathrm{~cm} / \mathrm{s}$, ou seja, valores maiores que os obtidos por Santos (2012) no mesmo aterro sanitário $\left(10^{-5} \mathrm{~cm} / \mathrm{s}\right)$ e por outros autores (RITTER et al., 2002; FRANCESCHET et al., 2005; TOZETTO, 2008).

Conforme Pinto (2006), o coeficiente de permeabilidade se relaciona com a estabilidade do solo porque a tensão efetiva (que comanda a resistência do solo) depende da pressão neutra, que, por sua vez, depende das tensões provocadas pela percolação da água. Para Kölsch e Bauer (2009), a presença de grandes volumes de água dentro do aterro reduz a pressão efetiva normal e a resistência ao cisalhamento. Por isso, projetos 
de recirculação de chorume em aterros sanitários precisam ser bem dimensionados e monitorados (JAFARI; STARK; MERRY, 2013).

Huvaj-Sarihan e Stark (2008), após retroanálise do rompimento de quatro aterros sanitários (Polônia, Turquia, Israel e Filipinas), apontaram entre as possíveis causas desses acidentes a presença elevada de água, tanto nos solos quanto nos resíduos. Os autores também citaram presença de resíduos da construção civil na camada de cobertura (algo também observado nessa pesquisa), presença de rachaduras no topo do aterro, acúmulo elevado de chorume dentro do maciço, taludes muito íngremes, falta de cobertura vegetal e de drenagem de águas pluviais, grande processo erosivo e bolsões de água no topo do aterro (algo também observado nessa pesquisa).

Conforme Peng et al. (2016), o aumento adicional da água no interior do aterro aumenta o peso dos resíduos sólidos, ocasionando grandes deformações do maciço e, consequentemente, deslizamentos da camada de cobertura. Para melhorar a resistência do solo de um aterro sanitário, Rong, Zhaogui e Tugen (2011) sugerem aumentar a espessura e a compactação da camada de cobertura e promover um bom sistema de drenagem de lixiviados e de águas pluviais, reduzindo a saturação do solo.

A rigidez de um solo de aterro sanitário também pode ser correlacionada com a presença ou ausência de fissuras/trincas. Conforme Lakshmikanta (2009), o processo de fissuramento se inicia quando as forças de tensão excedem a resistência à tração do solo, sendo que essa resistência é função do teor de umidade do solo e da sucção. Dessa maneira, segundo os autores, as fissuras podem constituir caminhos preferenciais à percolação da água da chuva e gases resultantes da biodegradação, aumentando a instabilidade da área. White et al. (2008) acrescentam que a rigidez não uniforme (em termos de valores) e a intensidade da referida tensão são os fatores que determinam o grau de fissuramento do solo.

Para Kölsch e Bauer (2009), o parâmetro que mais contribui para o aumento da estabilidade de um aterro sanitário é a densidade dos resíduos, por entenderem que uma alta densidade cria uma alta tensão normal, resultando em alta resistência ao cisalhamento. Além disso, complementam os autores, uma maior densidade pode resultar em menor permeabilidade, mantendo um equilíbrio hídrico adequado dentro do aterro.

\section{Conclusão}

Os aterros sanitários precisam de monitoramento geotécnico e ambiental durante sua vida útil, e por muitos anos após seu encerramento, para evitar acidentes (desmoronamento e explosões) e danos ambientais (contaminação do ar, dos solos e recursos hídricos superficiais e subterrâneos).

Em linhas gerais, a área estudada parece mais instável que estável, pois predominaram os pontos do solo classificados como fracamente rígidos. Tiveram grande influência sobre tal classificação os resultados medidos no talude. A presença de materiais grosseiros nos taludes (pedaços de madeira, pneus) contribuem para esses resultados. Cabe destacar que a estabilidade do aterro, como bem discutido ao longo do texto, não se restringe aos resultados do solo isoladamente.

A rigidez dos solos da camada de cobertura do aterro sanitário, diferentemente de outras obras citadas na literatura, tem significativa variabilidade em curtas distâncias, o que evidencia a necessidade de mais atenção e monitoramento geotécnico.

As correlações apresentadas neste artigo retratam a realidade de um aterro real, ou seja, em plena operação, e servirão para comparações com os resultados de diferentes experiências.

Alguns parâmetros, mesmo que não determinados nesta pesquisa, parecem ter forte influência sobre a rigidez do solo do aterro sanitário: compactação; pressão de líquidos e gases; balanço hídrico dos solos e resíduos; tipo de solo; histórico das tensões; trincas etc.

O Humboldt GeoGauge TM mostrou-se viável e adequado para a medição in situ da rigidez dos solos da camada de cobertura do aterro sanitário em questão, abrindo, inclusive, novas linhas de investigação para a engenharia sanitária e ambiental e, principalmente, para o controle geotécnico desses empreendimentos, o 
que não exclui a possibilidade de utilizá-lo concomitantemente a outros equipamentos e testes laboratoriais, os quais também necessitam de cuidado na interpretação dos resultados.

\section{Referências}

ABU-FARSAKH, M. et al. Evaluating the Stiffness Modulus of Highway Materials from the Geogauge Device. In: GEO JORDAN CONFERENCE, 2004, Irbid. Proceedings [...]. Irbid: American Society of Civil Engineers, 2004. p. 287-298. Disponível em: https://ascelibraryorg.ez11.periodicos.capes.gov.br/doi/ pdf/10.1061/40735\%28143\%2924. Acesso em: 22 abr. 2019.

ALBRIGHT, W. H. et al. Field perfomance of a compacted clay landfill final cover at a humid site. Journal of Geotechnical and Geoenvironmental Engineering, United States of America, v. 132, n. 11, p. 1393-1403, nov. 2006

ALSHIBLI, K. A. et al. Laboratory Evaluation of the Geogauge and Light Falling Weight Deflectometer as Construction Control Tools. Jornal of Materials in Civil Engineering, United States of America, v. 17, n. 5, p. 560-569, out. 2005. Disponível em: https://ascelibrary.org/doi/pdf/10.1061/\%28ASCE\%2908991561\%282005\%2917\%3A5\%28560\%29. Acesso em: 22 mar. 2019.

ALVES, A. K. Proposta de manual técnico de medidas preventivas e corretivas para aterros sanitários encerrados. 2010. 210 f. Dissertação (Mestrado em Engenharia Civil) - Universidade Federal de Uberlândia, Uberlândia, 2010.

BABU, G. L. S.; REDDY, K. R.; SRIVASTAVA, A. Influence of spatially variable geotechnical properties of MSW on stability of landfill slopes. Journal of Hazardous, Toxic, and Radioactive Waste, Bangalore, v. 18, n. 1, p. 27-37, jan. 2014. Disponível em: https://ascelibrary.org/doi/pdf/10.1061/\%28ASCE\%29 HZ.2153-5515.0000177. Acesso em 25 fev. 2019.

BORGATTO, A. V. A. Estudo das propriedades geomecânicas de resíduos sólidos urbanos prétratados. 2010. 306 f. Tese (Doutorado em Engenharia Civil) - Universidade Federal do Rio de Janeiro, Rio de Janeiro, 2010.

BOSCOV, M. E. G. Geotecnia ambiental. São Paulo: Oficina de Textos, 2008.

CATAPRETA, C. A. A. Comportamento de um aterro sanitário experimental: avaliação da influência do projeto, construção e operação. 2008. 316 f. Tese (Doutorado em Saneamento, Meio Ambiente e Recursos Hídricos) - Universidade Federal de Minas Gerais, Belo Horizonte, 2008.

CHEN, D. H. et. al. Evaluation of in situ resilient modulus testing techniques. In: GEOTECHNICAL SPECIAL PUBLICATION, 1999, Urbana-Champaign. Proceeding [...]. Urbana-Champaign: American Society of Civil Engineers, 1999. p. 1-11. Disponível em: http://www.hanmicorp.net/MFG/Humboldt/GeoGauge/GeoGauge-Reports/TXDOT\%20Report.pdf. Acesso em: 22 jan. 2019.

COLLINS, R.; CONNOR, B. The use of geofibers and synthetic fluids Kwigillingok Airport. Fairbanks: Alaska University Transportation Center, 2011.

CONDE, M. C. et al. Applicability of the Geogauge, P-FWD and DCP for compaction control.

In: INTERNATIONAL CONFERENCE ON SOIL MECHANICS AND GEOTECHNICAL ENGINEERING, 18. 2013, Paris. Proceedings [...]. Paris, 2013. p. 1263-1266. Disponível em: http://www.cfms-sols.org/sites/ default/files/Actes/1263-1266.pdf. Acesso em: 18 maio 2019.

CONDE, M. C.; CALDEIRA, L.; LOPES, M. G. Estudo das condições de aplicação do GeoGauge e do deflectômetro de impacto portátil no controlo da compactação. In: CONGRESSO NACIONAL DE GEOTECNIA, 12., 2010, Guimarães. Anais [...]. Guimarães: Sociedade Portuguesa 
de Geotecnia, 2010. p. 1767-1776. Disponível em: <http://repositorio.Inec.pt:8080/jspui/ bitstream/123456789/1000328/1/1767-1776_conde-estudo.pdf>. Acesso em: 17 maio 2019.

COSTA, C. M. C. Avaliação da fissuração por ressecamento em camadas de cobertura de aterros sanitários utilizando materiais alternativos. 2015. $181 \mathrm{f}$. Tese (Doutorado em Engenharia Civil e Ambiental) - Universidade de Brasília, Brasília, 2015.

EID, H. T. Shear strength of geosynthetic composite systems for design of landfill liner and cover slopes. Geotextiles and Geomembranes, Holland, v. 29, n. 3, p. 335-344, jun. 2011. Disponível em: https:// www.sciencedirect.com/science/article/pii/S0266114410001226?via\%3Dihub. Acesso em: 29 jul. 2019.

FENG, S. J.; GAO, L. Y. Seismic stability analyses for landfill cover systems under different seepage buildup conditions. Environmental Earth Sciences, Germany, v. 66, n. 1, p. 381-391, maio 2012. Disponível em: https://journals.sagepub.com/doi/10.1177/0734242X18757628. Acesso em: 25 maio 2019.

FORTUNATO, E. Renovação de Plataformas Ferroviárias: estudos relativos à capacidade de carga. 2005. 628 f. Tese (Doutorado em Engenharia Civil) - Universidade do Porto, Porto, 2005.

FRANCESCHET, M. et al. Estudo dos solos utilizados para a impermeabilização da camada de base e de cobertura de aterros sanitários de Santa Catarina. In: CONGRESSO BRASILEIRO DE ENGENHARIA SANITÁRIA E AMBIENTAL, 23, 2005, Campo Grande. Anais [...]. Campo Grande: Associação Brasileira de Engenharia Sanitária e Ambiental, 2005. Disponível em: http://www.bvsde.paho.org/bvsacd/abes23/III-092. pdf. Acesso em: 13 mar. 2019.

FREDLUND, D. G.; RAHARDJO, H. Soil mechanics for unsaturated soils. New York: John Wiley and Sons, 1993.

HOSSAIN, S.; HAQUE, A. Stability analyses of municipal solid waste landfills with decomposition. Geotechinical and Geological Engeneering, Holland, v. 27, n. 6, p. 659-666, dez. 2009. Disponível em: https://link.springer.com/content/pdf/10.1007\%2Fs10706-009-9265-0.pdf. Acesso em: 15 maio 2019.

HUMBOLDT MFG. CO. GeoGauge User Guide, Norridge. Version 4.1, 2007. Disponível em: https://www. mltest.com/PDF/H-4140_-_GeoGauge.pdf. Acesso em: 08 fev. 2019.

HUVAJ-SARIHAN, N.; STARK, T. D. Back-Analyses of Landfill Slope Failures. In: INTERNATIONAL CONFERENCE ON CASE HISTORIES IN GEOTECHNICAL ENGINEERING, 6., 2008, Arlington.

Proceedings[...]. Arligton: Missouri University of Science and Technology, 2008. p. 1-8. Disponível em: https://scholarsmine.mst.edu/cgi/viewcontent.cgi?article=2708\&context=icchge. Acesso em: 30 mar. 2019.

JAFARI, N. H.; STARK, T. D.; MERRY, S. The July 102000 Payatas Landfill Slope Failure. International Journal of Geo-Engineering, United Kingdom, v. 2, n. 3, p. 208-228. 2013. Disponível em: https://www. geocasehistoriesjournal.org/pub/article/view/IJGCH_2_3_3. Acesso em: 25 mar. 2019.

JIANGUO, L.; YONGFENG, N.; QINGZHONG, B. Analysis of structural stability in sanitary landfill. Chongqing Environmental Science, China, v.1, p. 62-66, fev. 2001.

JONES, D. R. V.; DIXON, N. Landfill lining stability and integrity: the role of waste settlement. Geotextiles and Geomembranes, Holland, v. 23, n. 1, p. 27-53, fev. 2005. Disponível em: https://www-sciencedirect. ez11.periodicos.capes.gov.br/science/article/pii/S0266114404000494. Acesso em: 26 maio 2019.

KOERNER, M. R.; HWU, B. L. Stability and tension considerations regarding cover soils on geomembrane lined slopes. Geotextiles and Geomembranes, Holland, v. 10, n. 4, p. 335-355, 1991. Disponível em: <https://www.sciencedirect.com/science/article/pii/026611449190010T?via\%3Dihub>. Acesso em: 24 ago. 2019. 
KÖLSCH, F.; BAUER, J. Static Stability of Landfills. In: INTERNATIONAL WASTE MANAGEMENT AND LANDFILL SYMPOSIUM, 12., 2009, Sardenha. Proceedings [...]. Sardenha: Internacional Waste Working Group - IWWG, 2009. p. 1-18. Disponível em: http://www.dr-koelsch.de/Sardinia_09_stability.pdf. Acesso em: 07 jul. 2019.

LAKSHMIKANTA, M. R. Experimental and theoretical analysis of cracking in drying soils. 2009. 390 f. Thesis (PhD Enginyeria del Terreny, Cartogràfica i Geofísica) - Universitat Politècnica de Catalunya, Barcelona, 2009. Disponível em: https://www.researchgate.net/publication/263046901_Experimental_and_ theoretical_analysis_of_cracking_in_drying_soils. Acesso em 23 abr. 2019.

LAW, H. J. et al. Maximizing landfill capacity by vertical expansion: a case study for an innovative waste management solution. In: ISWA WORLD CONGRESS, Vienna, 2013. Proceedings [...]. Vienna: International Solid Waste Association, 2013. Disponível em: https://pdfs.semanticscholar.org/0c7f/4a43ebdf8 fc1dba8571258b60a59ab4075ff.pdf. Acesso em: 25 maio 2019.

LENKE, L. R.; GORDON McKEEN, R.; GRUSH, M. P. Laboratory Evaluation of GeoGauge for Compaction Control. Transportation Research Record, United States of America, v. 1849, v. 1, p. 20-30, jan. 2003. Disponível em: https://journals.sagepub.com/doi/pdf/10.3141/1849-03. Acesso em: 05 abr. 2019.

LOPES, M. L.; GOMES, C. C. Geotechnical landfill monitoring - adaptations needed. Environmental Geotechnics, United Kingdom: ICE Publishing Ltd, 2013. p. 8-17. Disponível em: https://www. icevirtuallibrary.com/doi/pdf/10.1680/envgeo.13.00014. Acesso em: 26 fev. 2019.

MAIA, C. L.; SILVA, C. A. U.; BARROSO, S. H. A. Comparative analysis of resilience modules obtained in field with geogauge and those obtained in the laboratory. In: INTERNACIONAL SOCIETY FOR ASPHALT PAVEMENTS, 13., Fortaleza, 2018. Proceedings [...]. Fortaleza: ISAP, 2018. Disponível em: http:// icongresso.ikone.itarget.com.br/arquivos/trabalhos_completos/ikone/3/269_17022018_200313.pdf. Acesso em: 25 fev. 2019.

MEDEIROS, P. A. et al. Balanço hídrico em aterros de resíduos sólidos urbanos escala experimental de laboratório. In: CASTILHOS JÚNIOR, A. B. de, et al. (Org.). Alternativas de disposição de resíduos sólidos urbanos para pequenas comunidades (coletânea de trabalhos técnicos). Rio de Janeiro, ABES, 2002. p. 39-46. Disponível em: https://www.finep.gov.br/images/apoio-e-financiamento/historico-deprogramas/prosab/livrocompletofinal.pdf. Acesso em: 03 jul. 2019.

MERRY, S. M.; KAVAZANJIAN, E.; FRITZ, W. U. Reconnaissance of the July 10, 2000, payatas landfill failure. Journal of Performance of Constructed Facilities, United States of America, v. 19, n. 2, p. 100-107, maio, 2005. Disponível em: https://ascelibrary.org/doi/pdf/10.1061/\%28ASCE\%2908873828\%282005\%2919\%3A2\%28100\%29. Acesso em: 10 ago. 2019.

MOREIRA, C. A. et al. Caracterização geofísica em aterro de resíduos sólidos para avaliação de condicionantes de instabilidade geotécnica. Geologia USP - Serie Científica, São Paulo, v. 13, n. 1, p. 2534, maio 2013. Disponível em: http://www.revistas.usp.br/guspsc/article/view/54080/58010. Acesso em: 15 abr. 2019.

NELSON, C. R.; SONDAG, M. Comparison of the Humboldt GeoGauge with In-Place Quasi-Static Plate Load Tests. Minneapolis: CNA Consulting Engineers Report, 1999. Disponível em: http://hanmicorp. net/MFG/Humboldt/GeoGauge/GeoGauge--Reports/CNA\%20Plate\%20Load\%20Report.doc.pdf. Acesso em: 06 jun. 2019.

OLIVEIRA, D. A. F. Estabilidade de taludes de maciços de resíduos sólidos urbanos. 2002. $173 \mathrm{f}$. Dissertação (Mestrado em Geotecnia) - Universidade de Brasília, Brasília, 2002.

PENG, R. et al. Back-Analyses of landfill instability induced by high water level: case study of Shenzhen landfill. International Journal of Environmental Research and Public Health, Switzerland, v. 13, n. 1, 
p. 1-17, jan. 2016. Disponível em: https://www.researchgate.net/publication/290476345_Back-Analyses_ of_Landfill_Instability_Induced_by_High_Water_Level_Case_Study_of_Shenzhen_Landfill. Acesso em: 02 maio 2019.

PESTANA, R. M. M. Contribuição ao estudo do comportamento mecânico de resíduos de construção e demolição aplicados em estradas de baixo tráfego. 2008. 153 f. Dissertação (Mestrado em Engenharia Civil) - Universidade Técnica de Lisboa, Lisboa, 2008.

PINTO, C. S. Curso básico de mecânica dos solos em 16 aulas. São Paulo: Oficina de Textos, 2006.

QUINTA-FERREIRA, M. Ground stiffness evaluation using the Soil Stiffness Gauge (SSG). In: Lollino et al. Engineering geology for society and territory. Heidelberg: Springer, 2015. v. 6, p. 259-262. Disponível em: https://link.springer.com/chapter/10.1007/978-3-319-09060-3_42. Acesso em: 02 abr. 2019.

QUINTA-FERREIRA, M.; ANDRADE, P. S. Alguns resultados do Geogauge e do deflectómetro de impacto (FWD) na caracterização de pavimentos. In: CONGRESSO LUSO-BRASILEIRO DE GEOTECNIA, 4., 2008 p.161-168, Anais [...]. Coimbra: Associação Brasileira de Mecânica dos Solos e Engenharia Geotécnica (ABMS), 2008. p. 161-168.

REMÉDIO, F. H. Análise de estabilidade de taludes de aterro de resíduos sólidos utilizando parâmetros geotécnicos de propostas bibliográficas e correlações com $\mathbf{N}_{\mathrm{SPT}}$. 2014. $131 \mathrm{f}$. Dissertação (Mestrado em Geociências e Meio Ambiente) - Universidade Estadual Paulista, Atibaia, 2014.

RITTER, E. et al. Avaliação da permeabilidade da barreira de proteção da fundação do aterro sanitário de Piraí-RJ. In: SIMPÓSIO ÍTALO BRASILEIRO DE ENGENHARIA SANITÁRIA E AMBIENTAL, 6., 2002, Vitória. Anais [...]. Vitória: Associação Brasileira de Engenharia Sanitária e Ambiental, 2002. p. 1-6. Disponível em: https://docplayer.com.br/23017102-lii-023-avaliacao-da-permeabilidade-da-barreira-deprotecao-da-fundacao-do-aterro-sanitario-de-pirai.html. Acesso em: 07 abr. 2019.

RONG, F.; ZHAOGUI, G.; TUGEN, F. Analysis of stability and control in landfill sites expansion. In: PROCEDIA ENGINEERING. Holland: Elsevier, 2011. v. 24, p. 667-671. Disponível em: https://www. sciencedirect.com/science/article/pii/S1877705811055676. Acesso em: 18 maio 2019.

SANTOS, G. O. Avaliação do cultivo de gramíneas na superfície de aterro sanitário, com ênfase para a redução da emissão de metano e dióxido de carbono para a atmosfera. 2012. 313 f. Tese (Doutorado em Engenharia Civil) - Universidade Federal do Ceará, Fortaleza, 2012.

SARAVANAN, M. et al. Landfill interface study on liner member selection, stability assessment, and factor of safety predictions with seismic loading. In: MALAYSIA-JAPAN SYMPOSIUM ON GEOHAZARDS AND GEOENVIRONMENTAL ENGINEERING, 2., Bangi, 2004. Proceedings [...]. Bangi: Human Society for Sustainable Development, 2007. p. 1-15. Disponível em: https://www.researchgate.net/ publication/295856756_Landfill_interface_study_on_liner_selection_stability_assessment_and_factor_of_ safety_prediction_with_seismic_loading. Acesso em: 05 abr. 2019.

SCHULER, A. R. Análise do comportamento de um aterro municipal de resíduos sólidos urbanos instrumentado. 2010. 173 f. Dissertação (Mestrado em Engenharia Civil) - Universidade Federal do Rio de Janeiro, Rio de Janeiro, 2010.

SILVA, R. A. C da. Avaliação da evolução do comportamento geomecânico de aterros sanitários com base na realização de ensaios de laboratório e de campo. 2017. 138 f. Tese (Doutorado em Geologia) Universidade Federal do Ceará, Fortaleza, 2017.

SISTEMA NACIONAL DE INFORMAÇÕES SOBRE SANEAMENTO - SNIS. Diagnóstico do manejo de resíduos sólidos urbanos. Brasília: Ministério das Cidades, Secretaria Nacional de Saneamento Ambiental - SNSA, 2016. 
STRAUSS, M. Análise de estabilidade de talude do Aterro Sanitário da Zona Norte de Porto Alegre. 1998. 137 f. Dissertação (Mestrado em Engenharia Civil) - Universidade Federal do Rio Grande do Sul, Porto Alegre, 1998.

TOZETTO, C. M. Modelagem matemática de aterros sanitários com a simulação hidrológica da geração de lixiviado: estudo de caso do aterro sanitário de Curitiba. 2008. 156 f. Dissertação (Mestrado em Engenharia de Recursos Hídricos e Ambiental) - Universidade Federal do Paraná, Curitiba, 2008.

WHITE, D. J. et al. Fly ash soil stabilization for non-uniform subgrade soils. Tech Transfer Summaries, lowa State University, abr. 2005. Disponível em: https://core.ac.uk/download/pdf/76634784.pdf. Acesso em: 05 abr. 2019.

XU, Q.; TOLAYMANT, T.; TOWSEND, T. G. Impact of pressurized liquids addition on landfill slope stability. Journal of Geotechical and Geoenvironmental Engineering - ASCE, United States of America, v. 138, n. 4, p. 472-480, abr. 2012. Disponível em: https://ascelibrary.org/doi/pdf/10.1061/\%28ASCE\%29 GT.1943-5606.0000609. Acesso em: 20 abr. 2019.

YIN, Y. et al. Mechanism of the december 2015 catastrophic landslide at the Shenzhen landfill and controlling geotechnical risks of urbanization. Engineering, [s. I.], v. 2, n, 2, p. 230-249, jun. 2016. Disponível em: https://www.sciencedirect.com/science/article/pii/S209580991630950X. Acesso em: 25 abr. 2019.

\section{Sobre os autores}

\section{Samara Castro Freire}

Mestrado em andamento como bolsista do CNPQ. Graduação em Tecnologia em Gestão Ambiental pelo Instituto Federal de Educação Ciência e Tecnologia do Ceará (2017) - campus Fortaleza. Tem experiência em Ciências Ambientais, com ênfase em Gestão Ambiental, atuando principalmente nos seguintes temas: microbiologia ambiental (treinamentos de análises microbiológicas para alunos de graduação e pós-graduação da área ambiental), resíduos sólidos e aterros sanitários (monitoramento e diagnóstico do aproveitamento energético de resíduos e da geotecnia de aterros).

\section{Francisco Carlos Henrique Pio de Oliveira}

Graduação em andamento em Tecnologia em Gestão Ambiental pelo Instituto Federal de Educação Ciência e Tecnologia do Ceará (2016). Atualmente, é analista de limnologia e microbiologia do IFCE - campus Fortaleza.

\section{Eliana Dantas Ribeiro}

Graduação em Administração pela Universidade Estadual do Ceará (2013). Curso de Técnico em Edificações pelo Instituto Federal de Educação Ciência e Tecnologia do Ceará - campus Fortaleza (2015). Atualmente, é estagiário do Consórcio Metro Service. Tem experiência na área de Engenharia Civil.

\section{Roberto Antônio Cordeiro da Silva}

Doutorado em Geologia pela Universidade Federal do Ceará (2017). Graduação em Estudos Sociais pela UFC (1989). Graduação em Geografia pela UFC (2000). Mestrado em Geologia pela UFC (2004). Atualmente, é professor pleno i na Secretaria da Educação Básica e da Universidade Federal do Ceará. Tem experiência na área de Geociências, com ênfase em geologia ambiental, atuando principalmente nos seguintes temas: solos, lagoa, erosão, geotécnica e oxigênio.

\section{Gemmelle Oliveira Santos}

Doutorado em Engenharia Civil (linha: Saneamento ambiental) pela Universidade Federal do Ceará (2019). Mestrado em Saúde Pública (linha: Produção, ambiente e saúde) pela UFC (2008). Especialização em Educação Ambiental pela Universidade Estadual do Ceará (2007). Graduação Tecnológica em Gestão Ambiental pelo IFCE (2006). Professor efetivo do IFCE (campus Fortaleza) no curso de graduação em Tecnologia em Gestão Ambiental, no bacharelado em Engenharia Civil e no mestrado em Tecnologia em Gestão Ambiental (PGTGA). Atualmente, desenvolve atividades na área de gestão e gerenciamento de resíduos sólidos urbanos. 